\title{
Minimally Invasive Aortic Valve Replacement with Partial Lower Sternotomy
}

\author{
Keisuke Morimoto ${ }^{1,2^{*}}$, Shigeto Miyasaka ${ }^{1}$, Suguru Shiraya ${ }^{1}$, Futoshi Kobayashi ${ }^{1}$ \\ ${ }^{1}$ Department of Cardiovascular Surgery, Tottori Prefectural Central Hospital, Tottori, Japan \\ ${ }^{2}$ Department of Cardiovascular Surgery, Sanin Rosai Hospital, Yonago, Japan \\ Email: ${ }^{\star}$ morimoto@saninh.johas.go.jp
}

How to cite this paper: Morimoto, K. Miyasaka, S., Shiraya, S. and Kobayashi, F. (2018) Minimally Invasive Aortic Valve Replacement with Partial Lower Sternotomy. World Journal of Cardiovascular Surgery, 8, 103-110.

https://doi.org/10.4236/wjcs.2018.86009

Received: April 2, 2018

Accepted: June 12, 2018

Published: June 15, 2018

Copyright () 2018 by authors and Scientific Research Publishing Inc. This work is licensed under the Creative Commons Attribution International License (CC BY 4.0).

http://creativecommons.org/licenses/by/4.0/

\begin{abstract}
Objectives: Due to recent spread of minimally invasive surgery, the demand for minimally invasive cardiac surgery (MICS) is increasing. We investigate the usefulness of minimally invasive aortic valve replacement (MICS-AVR) which was performed in our hospital. Methods: Of 63 consecutive patients undergone an isolated aortic valve replacement (AVR), 16 patients underwent MICS-AVR with partial lower sternotomy (M group) and 47 patients underwent AVR with median full sternotomy (C group). We compared the two groups in a retrospective study. Results: No significant difference was found in the surgical and perioperative-related factors between the two groups. However, the average of aortic cross-clamping time was longer, and intensive and high care unit stay was shorter in the $\mathrm{M}$ group. A tendency to decrease blood transfusion was observed in the $\mathrm{M}$ group. There was no hospital death in all patients. The mean follow-up period was $29 \pm 15$ months. There was no significant difference between the two groups in the survival rate, and the 5-year survival rates were $88.9 \%$ in the M group and $85.9 \%$ in the $\mathrm{C}$ group. Conclusion: It was suggested that the MICS-AVR could be safe and useful procedure in AVR.
\end{abstract}

\section{Keywords}

Minimally Invasive Cardiac Surgery (MICS), Aortic Valve Replacement (AVR), Partial Sternotomy

\section{Introduction}

Aortic valve replacement (AVR) as a surgical treatment for aortic valve disease has been performed for many years by the median sternotomy approach. Since the 1990s, minimally invasive cardiac surgery (MICS) has been undertaken by 
the small incision. The surgical technique with a right parasternal incision was reported as the first minimally invasive aortic valve replacement (MICS-AVR) performed by Cosgrove et al. in 1996 [1]. Initially the evaluation of MICS in Japan has been low and it has not spread very much. However, due to the recent spread of minimally invasive surgical operations and the progress of surgical instruments, the demand for MICS is increasing. Some surgical approaches have been performed for MICS-AVR, and the approach with a partial upper sternotomy [2] is commonly used. We have started MICS for cardiac valve diseases since 2010 in our hospital, and we have adopted an approach via a partial lower sternotomy. In this study, we investigated MICS-AVR with the partial lower sternotomy which was performed in our hospital.

\section{Methods}

\subsection{Patients}

From February 2011 to October 2015, 102 consecutive patients underwent AVR in our hospital. Of these patients, 67 had isolated AVR. Among them, 2 of redo surgery, 1 of emergency operation and 1 of active infectious endocarditis were excluded. In the analysis, there were a total of 63 patients who underwent isolated AVR. In these patients, we compared them separately into 16 patients with MICS-AVR (M group) and 47 patients with median sternotomy (C group).

The patient demographic data were comparable between the two groups as shown in Table 1. The comparable demographics were age, gender, New York Heart Association (NYHA) functional classification, left ventricular ejection fraction (LVEF), the rate of patients of hypertension, diabetes mellitus, chronic obstructive pulmonary disease (COPD), hemodialysis, presence of smoking, aortic stenosis (AS), and serum creatinine ( $\mathrm{Cr}$ ) value. The decision of whether patients underwent MICS-AVR or full sternotomy was predominantly made by the staff surgeons after the preoperative heart team conference, and the informed consent for the patients was obtained. In addition, patients with a medical history of pacemaker implantation and moderate or higher arteriosclerotic changes judged by preoperative enhanced CT examination were not adapted for MICS-AVR, and these patients underwent AVR by median sternotomy. Furthermore, MICS-AVR was performed by the two senior medical staff.

\subsection{Operative Management and Surgical Procedure}

MICS-AVR was performed with an about $10 \mathrm{~cm}$ skin incision in the median longitudinal direction of the lower sternum, and the partial sternotomy was performed with an inverted L-shaped sternotomy incision from the second right intercostal space of the sternum to the xiphoid. In the $\mathrm{C}$ group, the superior and inferior vena cava and the ascending aorta were cannulated separately to institute cardiopulmonary bypass (CPB). In the $\mathrm{M}$ group, the superior vena cava and inferior vena cava through right femoral vein and the femoral artery were cannulated separately to institute the CPB. All patients of both groups were 
Table 1. Patient demographics.

\begin{tabular}{cccc}
\hline $\begin{array}{c}\text { Variables, mean } \pm \text { SD } \\
\text { or \% }\end{array}$ & $\begin{array}{c}\text { Group M } \\
(\mathrm{n}=16)\end{array}$ & $\begin{array}{c}\text { Group C } \\
(\mathrm{n}=47)\end{array}$ & $p$ value \\
\hline Age, year & $72.1 \pm 9.7$ & $74.4 \pm 9.6$ & 0.425 \\
Sex, male \% & 50.0 & 58.5 & 0.576 \\
NYHA classification & $2.1 \pm 0.4$ & $2.1 \pm 0.6$ & 0.925 \\
LVEF (\%) & $62.1 \pm 10.8$ & $62.1 \pm 14.5$ & 0.988 \\
Hypertension (\%) & 25.0 & 48.8 & 0.065 \\
Diabetes Mellitus (\%) & 25.0 & 22.0 & 0.963 \\
COPD (\%) & 6.3 & 12.2 & 0.468 \\
Hemodialysis (\%) & 12.5 & 9.8 & 0.781 \\
Smoking (\%) & 18.9 & 14.6 & 0.724 \\
Creatinine (mg/dl) & $1.5 \pm 2.3$ & $1.7 \pm 2.5$ & 0.790 \\
AS (\%) & 81.3 & 85.4 & 0.887 \\
\hline
\end{tabular}

NYHA, New York Heart Association; LVEF, left ventricular ejection fraction; COPD, chronic obstructive pulmonary disease; AS, Aortic valve stenosis.

performed AVR under the cardiac arrest using antegrade and retrograde myocardial protection with cold blood cardioplegia, and a left cardiac venting catheter was inserted through the right upper pulmonary vein. Regarding to the AVR, both patients routinely underwent the procedure by ascending aorti incision.

\subsection{Follow-Up and Statistics}

Follow-up was basically obtained by regular outpatient contact. Continuous variables are expressed as the mean \pm standard deviation. Comparisons of data between the two groups were performed using $t$ test for the continuous variables, Fisher's exact test for the ratios, and Mann-Whitney's $U$ test for the categorical variables, respectively. The survival curves were obtained by Kaplan-Meier method and the Log-rank test was performed. A $p$ value of less than 0.05 was considered significant. All statistical analyses were performed using SPSS version 20.0 .

\section{Results}

The mean age of patients was $72.1 \pm 9.7$ and $74.4 \pm 9.6$ years in M group and C group, respectively, and they were not significantly different. There was no significant difference in the ratio of sex between the two groups. There were no significant differences between the two groups in NYHA functional classification, LVEF and serum $\mathrm{Cr}$ value. And there were no significant differences between the two groups in the rate of patients of hypertension, diabetes mellitus, COPD, hemodialysis, presence of smoking and AS. These preoperative comparisons of patient characteristics between the two groups are showed in Table 1.

Operation time, total CPB time and aortic cross-clamp time were examined as intraoperative factors of the patients (Table 2). There were no significant 
Table 2. Operative data.

\begin{tabular}{cccc}
\hline & Group M & Group C & Group C \\
\hline Operation time (min) & $279 \pm 23$ & $268 \pm 42$ & 0.224 \\
CPB time (min) & $157 \pm 21$ & $148 \pm 27$ & 0.237 \\
AoXCl time (min) & $94 \pm 12$ & $87 \pm 17$ & 0.082 \\
\hline
\end{tabular}

$\mathrm{CPB}$, cardiopulmonary bypass; $\mathrm{AoXCl}$, aortic cross-clamp.

differences in the intraoperative factors between the two groups. However, the average values of the $M$ group were longer than those of the $C$ group, and there was a tendency to prolong aortic cross-clamp time in the $\mathrm{M}$ group.

The comparisons of perioperative factors and complications between the two groups are showed in Table 3. There were no significant differences between the two groups in postoperative intubation time, intensive and high care unit (ICU/HCU) stay, and postoperative hospital stay. The average durations of the $\mathrm{M}$ group were shorter than those of the $\mathrm{C}$ group, and there was a tendency to shorten ICU/HCU stay in the M group. There was no significant difference between the two groups in the intraoperative and postoperative transfusion volume of erythrocyte; however there was a tendency to decrease the transfusion in the $\mathrm{M}$ group. There was no significant difference between the two groups in the incidence of perioperative arrhythmia. In the perioperative complications (Table $3)$, sepsis in 1 (6.3\%) in the $\mathrm{M}$ group, reoperation due to postoperative bleeding in $1(2.4 \%)$ in the $\mathrm{C}$ group, cerebral infarction in 2 in the two groups, and deep wound infection in 2 in the two groups. There was no significant difference in the incidence of these perioperative complications between the two groups. There were no operative death and hospital death in each group.

Figure 1 shows postoperative survival curves of the two groups. The mean follow-up period was $29 \pm 15$ months. There was no significant difference between the two groups in the survival rate, and the 5-year survival rates were $88.9 \%$ in the $\mathrm{M}$ group and $85.9 \%$ in the $\mathrm{C}$ group. Mid-term mortality during the postoperative follow-up was 3 in all patients, including 1 aspiration pneumonia in the group $\mathrm{M}, 1$ heart failure due to left ventricular dysfunction in the group C, and 1 cancer death due to lung cancer.

\section{Discussion}

While being required to minimally invasive surgery in each surgical field, it is positioned as MICS in cardiac surgery. MICS-AVR was reported by Cosgrove et al. in 1996 [1]. After that, it was evaluated as leading to reduction of the ventilator wearing time, hospital stay, perioperative bleeding volume and transfusion [3] [4] [5] [6]. Some reports have not obtained results that MICS-AVR has advantages [7] [8], while the meta-analysis shows the superiority of MICS-AVR [4]. On the other hand, MICS-AVR causes extension of aortic cross-clamp time and $\mathrm{CPB}$ time due to technical difficulty in small surgical field [4]. In recent 
Table 3. Postoperative data.

\begin{tabular}{cccc}
\hline & Group M & Group C & $p$ value \\
\hline Intubation time (h) & $10.9 \pm 10.4$ & $14.9 \pm 17.8$ & 0.300 \\
Transfusion (unit & $4.8 \pm 3.1$ & $6.6 \pm 4.8$ & 0.095 \\
ICU/HCU stay (day) & $3.8 \pm 1.6$ & $5.1 \pm 4.2$ & 0.099 \\
Hospital stay (day) & $18.4 \pm 7.4$ & $22.1 \pm 12.3$ & 0.172 \\
Arrhythmia (\%) & 12.5 & 17.1 & 0.663 \\
Sepsis (\%) & 6.3 & 0 & 0.333 \\
Reoperation for bleeding (\%) & 0 & 2.4 & 0.323 \\
Cerebral infarction (\%) & 6.3 & 2.4 & 0.576 \\
Deep wound infection (\%) & 6.3 & 2.4 & 0.576 \\
Perioperative death (\%) & 0 & 0 & 1.000 \\
Hospital death (\%) & 0 & 0 & 1.000 \\
\hline
\end{tabular}

ICU/HCU, intensive and high care unit.

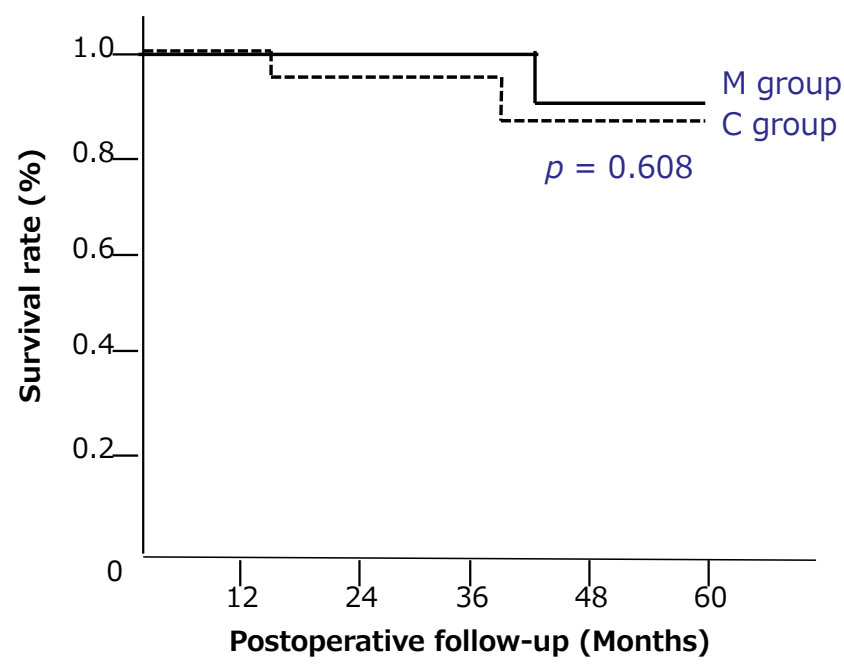

Figure 1. Postoperative survival curves.

years, some reports [9] [10] show that the advances of technical skills and devices used for MICS reduced the operation time. Lehmann et al. [2] reported the long-term results after MICS-AVR are good, however the advantages of MICS-AVR have not been clarified in Japan. In our study, although the number of patients was small and there was no significant difference between MICS-AVR and conventional AVR with full sternotomy in the clinical data, there was a tendency to extend the aortic cross-clamp time, reduce the transfusion volume, and shorten the period of ICU stay in the patients undergoing MICS-AVR as with the results reported by the households. In addition, the good results were obtained not only in the perioperative results but also in the mid-term results in the both groups in our study, and it seems that there was no problem in the surgical indication and the selection of the operation. 
Surgical approaches for MICS-AVR are via a partial upper sternotomy [2], a right intercostal small thoracotomy [11] and a partial lower sternotomy, etc. We emphasize a secure retrograde cardioplegia with direct placement of cannula using pursestring suture within coronary sinus, and we perform MICS-AVR via a partial lower sternotomy with the myocardial protection. In regard to the $\mathrm{CPB}$ in lower sternotomy approach, a peripheral artery such as a femoral artery is often selected as an arterial perfusion route because the cannulation in ascending aorta is anatomically difficult. Although a thromboembolism is a concern in the blood delivery from the femoral artery, it is considered safe if it is confirmed that there is no arteriosclerosis finding in a preoperative enhanced CT examination. Perioperative cerebral infarction in our study was observed for MICS-AVR in 1 patient and also for AVR with full sternotomy in 1 patient. The patient with MICS-AVR complicated by perioperative cerebral infarction had the onset on 2nd postoperative day. We consider that the cause of the perioperative cerebral infarction is not derived from the blood delivery from the femoral artery. In addition, MICS-AVR via a partial lower sternotomy has an advantage in preventing air embolism compared to other MICS-AVR with small incision because we can handle the left ventricle directly by the procedure.

Multiple studies reported longer aortic cross-clamp time in MICS, however, they did not show to increase the rate of related adverse effects such as perioperative myocardial infarction or low cardiac output syndrome in MICS [4] [5] [11] [12] [13]. In our experience, aortic cross-clamp time was slightly prolonged in MICS-AVR. However, there was no perioperative myocardial infarction and requiring mechanical assistance.

Glauber et al. [11] performed a propensity matched analysis comparing MICS-AVR to conventional AVR. They used a right anterior mini-thoracotomy approach in MICS-AVR. And they demonstrated a lower incidence of postoperative atrial fibrillation and blood transfusion, as well as shorter ventilation times and hospital stays in MICS patients with no difference in hospital mortality rates. In our study, there were no statistically significant differences between the two groups in these perioperative parameters, however the same trend as their research results was found.

Merk et al. [13] compared early and long-term outcomes of MICS-AVR to conventional AVR in patients undergoing isolated bioprosthetic AVR. After a propensity matched analysis, there were no clinically significant differences in preoperative variables. Their study had a significantly reduced in-hospital and long-term mortality rate in MICS-AVR patients. Our results show that there was no hospital death in all patients to be compared and the mid-term mortality rates were similarly low in both groups, therefore they did not lead to a significant difference. MICS-AVR is technically difficult because of the narrow surgical field compared to conventional AVR. It is important to perform MICS-AVR after becoming familiar with conventional AVR. We limited the operators of MICS-AVR to the two superiors in our facility. The operator selection may in- 
fluence the results such as aortic cross-clamp time and operation time in our study. And the limitation of this study is the fact that it is a retrospective, small number and single institution experience. In addition, our results are not enough to produce statistically significant differences. Based on these study limitations, we will make future investigations.

MICS-AVR with partial sternotomy is regarded as one of the approaches to aortic valve surgery, and is expected to change with the spread of new devices, sutureless and rapid deployment aortic valves in the near future. It is important that MICS-AVR can be performed safely and effectively, and the early and long-term results are acceptable.

\section{Conclusion}

We conclude that MICS-AVR with partial lower sternotomy can be performed safety and effectively, and the early and mid-term results are acceptable.

\section{Funding Statement}

There is no funding to declare.

\section{Conflicts of Interest Statement}

There is no conflict of interest to declare.

\section{References}

[1] Cosgrove III, D.M. and Sabik, J.F. (1996) Minimally Invasive Approach for Aortic Valve Operations. The Annals of Thoracic Surgery, 62, 596-597. https://doi.org/10.1016/0003-4975(96)00418-3

[2] Lehmann, S., Merk, D.R., Etz, C.D., Seeburger, J., Schroeter, T., Oberbach, A., et al. (2015) Minimally Invasive Aortic Valve Replacement: The Leipzig Experience. Annals of Cardiothoracic Surgery, 4, 49-56.

[3] Mihaljevic, T., Cohn, L.H., Unic, D., Aranki, S.F., Couper, G.S. and Byrne, J.G. (2004) One Thousand Minimally Invasive Valve Operations: Early and Late Results. Annals of Surgery, 240, 529-534. https://doi.org/10.1097/01.sla.0000137141.55267.47

[4] Bonacchi, M., Prifti, E., Giunti, G., Frati, G. and Sani, G. (2002) Does Ministernotomy Improve Postoperative Outcome in Aortic Valve Operation? A Prospective Randomized Study. The Annals of Thoracic Surgery, 73, 460-465. https://doi.org/10.1016/S0003-4975(01)03402-6

[5] Tabata, M., Umakanthan, R., Cohn, L.H., Bolman III, R.M., Shekar, P.S., Chen, F.Y., et al. (2008) Early and Late Outcomes of 1000 Minimally Invasive Aortic Valve Op erations. European Journal of Cardio-Thoracic Surgery, 33, 537-541. https://doi.org/10.1016/j.ejcts.2007.12.037

[6] Doll, N., Borger, M.A., Hain, J., Bucerius, J., Walther, T., Gummert, J.F., et al. (2002) Minimal Access Aortic Valve Replacement: Effects on Morbidity and Resource Utilization. The Annals of Thoracic Surgery, 74, 1318-1822. https://doi.org/10.1016/S0003-4975(02)03911-5

[7] Christiansen, S., Stypmann, J., Tjan, T.D., Wichter, T., Van Aken, H., Scheld, H.H., 
et al. (1999) Minimally-Invasive versus Conventional Aortic Valve Replacement-Perioperative Course and Mid-Term Results. European Journal of Cardio-Thoracic Surgery, 16, 647-652. https://doi.org/10.1016/S1010-7940(99)00333-4

[8] Detter, C., Deuse, T., Boehm, D.H., Reichenspumer, H. and Reichart, B. (2002) Midterm Results and Quality of Life after Minimally Invasive vs. Conventional Aortic Valve Replacement. Journal of Thoracic and Cardiovascular Surgery, 50, 337-341. https://doi.org/10.1055/s-2002-35743

[9] Kocher, A.A., Laufer, G., Haverich, A., Shrestha, M., Walther, T., Misfeld, M., et al. (2013) One-Year Outcomes of the Surgical Treatment of Aortic Stenosis with a Next Generation Surgical Aortic Valve (TRITON) Trial: A Prospective Multicenter Study of Rapid-Deployment Aortic Valve Replacement with the EDWARDS INTUITY Valve System. Journal of Thoracic and Cardiovascular Surgery, 145, 110-115. https://doi.org/10.1016/j.jtcvs.2012.07.108

[10] Folliguet, T.A., Laborde, F., Zannis, K., Ghorayeb, G., Haverich, A. and Shrestha, M. (2012) Sutureless Perceval Aortic Valve Replacement: Results of Two European Centers. The Annals of Thoracic Surgery, 93, 1483-1488.

https://doi.org/10.1016/j.athoracsur.2012.01.071

[11] Glauber, M., Miceli, A., Gilmanov, D., Ferrarini, M., Bevilacqua, S., Farneti, P.A., et al. (2013) Right Anterior Minithoracotomy versus Conventional Aortic Valve Replacement: A Propensity Score Matched Study. Journal of Thoracic and Cardiovascular Surgery, 145, 1222-1226. https://doi.org/10.1016/j.jtcvs.2012.03.064

[12] Murtuza, B., Pepper, J.R., Stanbridge, R.D., Jones, C., Rao, C., Darzi, A., et al. (2008) Minimal Access Aortic Valve Replacement: Is It Worth It? The Annals of Thoracic Surgery, 85, 1121-1131. https://doi.org/10.1016/j.athoracsur.2007.09.038

[13] Merk, D.R., Lehmann, S., Holzhey, D.M., Dohmen, P., Candolfi, P., Misfeld, M., et al. (2015) Minimal Invasive Aortic Valve Replacement Surgery Is Associated with Improved Survival: A Propensity-Matched Comparison. European Journal of Cardio-Thoracic Surgery, 47, 11-17. https://doi.org/10.1093/ejcts/ezu068 